\title{
Paternal inheritance in parthenogenetic forms of the planarian Schmidtea polychroa
}

\author{
TG D'Souza, RD Schulte, H Schulenburg and NK Michiels \\ Animal Evolutionary Ecology, Zoological Institute, University of Tuebingen, Auf der Morgenstelle 28, Tuebingen D-72076, Germany
}

\begin{abstract}
Parthenogenesis usually includes clonal inheritance, which is thought to increase the risk of the clonal populations' extinction. Yet many parthenogenetic organisms appear to have survived for extended periods. A possible explanation is that parthenogens occasionally reproduce through sex-like processes. Although there is indirect evidence for occasional sex, the underlying mechanisms are currently unknown. In the present study, we examined sex-like processes in the planarian flatworm Schmidtea (Dugesia) polychroa. Parthenogenetic forms of this species are simultaneous hermaphrodites that require sperm to trigger embryogenesis, whereas
\end{abstract}

paternal genetic material is usually excluded from the oocyte (sperm-dependent parthenogenesis). Based on a comparison of parents and offspring, using highly polymorphic microsatellites, we demonstrate the incorporation of paternal alleles in about $5 \%$ of the offspring. We detected two distinct processes: chromosome addition and chromosome displacement. Such rare sexual processes may explain the long-term persistence of the many purely parthenogenetic populations of $S$. polychroa in northern Europe.

Heredity (2006) 97, 97-101. doi:10.1038/sj.hdy.6800841; published online 24 May 2006

Keywords: evolution of sex; recombination; microsatellites; parthenogenesis; occasional sex; polyploidy

\section{Introduction}

Most types of parthenogenesis involve the production of clonal, genetically identical offspring as meiosis and karyogamy are usually absent. At the population level, amictic parthenogenesis reduces genetic variation, which is disadvantageous under nonequilibrium conditions (West et al, 1999; Otto and Lenormand, 2002). Continuous generation of new genetic variants is therefore essential for the persistence of parthenogenetic populations (Hurst and Peck, 1996; West et al, 1999; Schön et al, 2000). Such new variants may result from (1) the repeated origin of asexual lineages from sexual ancestors, (2) mutations or (3) occasional sexual recombination (Parker, 1979). Additionally, new clonal lineages may result from interspecific hybridization (eg Lamatsch et al, 2000; Lampert et al, 2005; Gomez-Zurita et al, 2006). The recurrent production of asexual lineages from sexuals is only possible if sexuals and asexuals coexist. In contrast, mutations and occasional sexual recombination may also occur in purely parthenogenetic populations. In this case, the sexual events, although rare, are suggested to be the most efficient mechanism to prevent extinction (Hurst and Peck, 1996).

Here, we prove the occurrence of rare sexual processes by analyzing paternal inheritance in parthenogens of the planarian flatworm Schmidtea polychroa. To our knowledge, this is the first study of its kind in parthenogenetic animals. S. polychroa is a simultaneous hermaphrodite incapable of self-fertilization. Parthenogenetic forms are polyploid, usually triploid $(3 x=12)$, rarely tetraploid $(4 x=16)$. They are sperm-dependent, that is, allosperm is

Correspondence: TG D'Souza, Animal Evolutionary Ecology, Zoological Institute, University of Tuebingen, Auf der Morgenstelle 28, Tuebingen D-72076, Germany. E-mail: thomas.dsouza@uni-tuebingen.de Received 24 January 2006; accepted 13 April 2006; published online 24 May 2006 needed to trigger zygote division and embryo development. Because parthenogenetic S. polychroa are also hermaphroditic, they produce polyploid, parthenogenetic eggs as well as haploid sperm (Benazzi Lentati, 1970). During spermatogenesis, one chromosome set (two in tetraploids) is eliminated. Subsequently, the resulting diploid spermatocytes undergo normal meiosis with haploid, fertile spermatozoa as end products (Benazzi Lentati, 1970; Storhas et al, 2000). Consequently, parthenogens can reproduce in the absence of diploid, sexual conspecifics, which is the rule in large parts of central and western Europe (Beukeboom et al, 1996; Pongratz et al, 2003). In order to achieve this, parthenogens mate and exchange sperm reciprocally (Michiels and Kuhl, 2003). Sperm-dependent parthenogens typically show no paternal genetic contribution as paternal chromosomes are expelled from the zygote after syngamy (Figure 1a). In a previous study of a purely parthenogenetic population, we nevertheless found substantial genetic differences between parents and offspring, strongly indicating the occurrence of sexual processes (D'Souza et al, 2004). However, this study was restricted to 'mother'-offspring comparisons in which the putative sperm contributors were unknown. As a result, it did not provide formal proof of paternal inheritance (D'Souza et al, 2004).

In the present study, we specifically crossed individuals with known genotypes. Using four highly polymorphic microsatellite loci, we compared genotypes of both adults and offspring to examine the occurrence of paternal inheritance.

\section{Materials and methods}

\section{Collection and culture}

We collected adult individuals from the same lake and location as in the previous study (Wartaweil at lake 
98

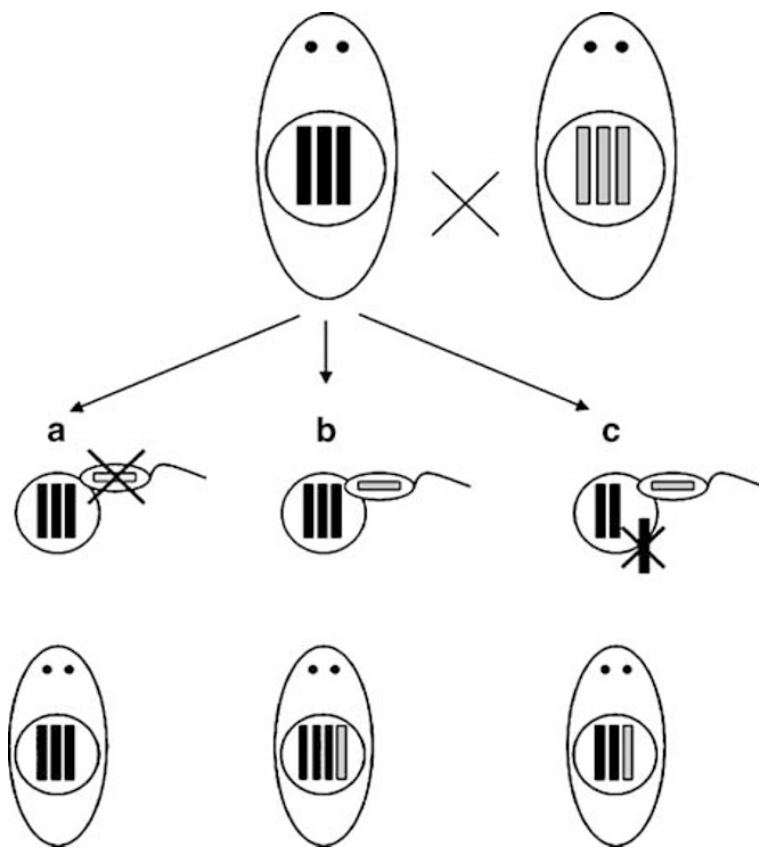

Figure 1 Paternal inheritance in parthenogenetic S. polychroa. Black bars indicate maternal and gray bars paternal chromosome sets. (a) Sperm-dependent parthenogenesis: Haploid sperm is used to trigger embryo development without paternal, genetic contribution to offspring. (b) Chromosome addition: haploid sperm fuses with polyploid egg, leading to an increase in ploidy (eg $3 x \rightarrow 4 x$ ). (c) Chromosome displacement: haploid sperm fuses with polyploid egg and one maternal chromosome set is excluded from the zygote.

Ammersee, Bavaria, Germany). This population consists exclusively of parthenogens (Beukeboom et al, 1996; Pongratz et al, 2003). Individuals were 'virginized' by decapitation, which removes the sperm storage organs. Head tissue was then prepared and fixed for karyology and DNA extraction. Individuals regenerated within $\sim 1$ month and were kept isolated under constant conditions ( $16^{\circ} \mathrm{C}$ and $12: 12 \mathrm{~h}$ dark:light cycle).

\section{Karyology}

Adult individuals were karyotyped using a modified protocol of Redi et al (1982). For this, partially regenerated individuals were treated with colchicine to arrest mitosis in the metaphase. Chromosomes were then counted using phase contrast microscopy (Beukeboom et al, 1996).

\section{Microsatellites}

Four highly polymorphic, trinucleotide microsatellites (SpATT7, SpATT9, SpATT12, SpATT20) were used for genetic analysis (Ramachandran et al, 1997; Pongratz et al, 2001). Information on DNA isolation, polymerase chain reaction (PCR), fragment analysis and the primers for loci SpATT12 and SpATT20 are given in Pongratz et al (2001) and D'Souza et al (2004). New primers and PCR profiles were used for loci SpATT7 (SpATT7F: 5'-GGCTC CTTTGTGTGGTTTGTA, SpATT7R: 5'-CCACCTGATGA TGACAGGAAA; profile: $2 \mathrm{~min} 95^{\circ} \mathrm{C}, 35$ cycles with $20 \mathrm{~s}$ $95^{\circ} \mathrm{C}, 1 \mathrm{~min}$ touchdown from 60 to $50^{\circ} \mathrm{C}, 1 \mathrm{~min} 72^{\circ} \mathrm{C}$ and a final $5 \mathrm{~min} 72^{\circ} \mathrm{C}$ ) and SpATT9 (SpATT9F: $5^{\prime}$-TGGGGAAG AAGAACATTGCTA, SpATT9R: 5'-AAATAAYCTGTGK GGAAAT; profile: $2 \mathrm{~min} 95^{\circ} \mathrm{C}$, 35 cycles with $20 \mathrm{~s} 95^{\circ} \mathrm{C}$,
$1 \min 60^{\circ} \mathrm{C}$, $1 \min 72^{\circ} \mathrm{C}$ ). For $S p A T T 9$, the number of alleles exceeded the ploidy level (up to seven alleles in triploid individuals), indicating that this microsatellite locus is present in at least three copies within the genome. Importantly, the presence of several loci does not preclude detection of paternal alleles among offspring. Loci SpATT12 and SpATT20 are not linked (Pongratz et al, 2001), whereas no information about the linkage of SpATT7 and SpATT9 is currently available.

\section{Crosses and paternity analysis}

For the crossing experiment, we focused on 20 randomly chosen individuals, which were genotyped at all four microsatellite loci prior crosses and kept in pairs for 1 week. All individuals differed in allele composition and therefore belonged to different clonal lineages. Partners within each pair shared no more than one allele per locus. Furthermore, at least one locus had no alleles in common.

As S. polychroa is hermaphroditic and sperm donation is generally bidirectional (Michiels and Streng, 1998; Michiels and Kuhl, 2003), all 20 individuals could potentially produce offspring. However, only 11 individuals produced fertile cocoons after separation. Cocoon production was observed in the following 23 weeks. All hatched offspring $(n=381)$ were genotyped with the two most polymorphic microsatellite markers (SpATT12, SpATT20). Genotyping was complemented with the two additional markers (SpATT7, SpATT9) when the first analysis revealed genetic differences between mother and offspring $(n=21)$. Karyotypes of offspring were indirectly determined using information of maternal ploidy level and inferred offspring microsatellite allele number. This has been proven to be a reliable approach in S. polychroa because at least loci SpATT12 and SpATT20 are unlinked (Pongratz et al, 2001) and have usually high individual heterozygosity (unpublished data).

\section{Results}

In 18 out of 381 offspring, we obtained unequivocal evidence for paternal alleles at two or more microsatellite loci (see Supplementary Information for details). Three more cases revealed mother-offspring differences at only one locus. Two of these showed loss of one maternal allele (offspring D4 and K1; Supplementary Information), whereas in the third case one maternal allele was missing and a new allele non-parental allele was found (offspring D5). In total, five out of 11 fertile individuals produced clonal offspring only, whereas evidence for paternal inheritance was found in the six remaining families. Within these six families, the proportion of offspring with paternal alleles ranged from 0.016 to 0.250 (Table 1).

The data indicate two alternative pathways for the incorporation of paternal alleles: (i) addition of paternal alleles $(n=10)$ and (ii) addition of paternal alleles accompanied by loss of maternal alleles $(n=8)$ (Figure $1 \mathrm{~b}$ and $\mathrm{c}$, and Table 2). The common characteristic of both pathways is the incorporation of maximally one paternal allele per locus (except the supernumerary alleles of SpATT9). However, they differ in maternal inheritance. While in the first pathway all maternal alleles were present in the offspring, in the second not all maternal alleles were inherited. These processes result in recom- 
bined offspring with more alleles than the maternal individual (addition) or with the same number of alleles as the maternal type (displacement).

Incorporation of paternal alleles was not always detectable at all four loci, but in all these cases the lack of observable differences could be explained by identical maternal and paternal alleles at a particular locus, or a single mutation in one of the paternal alleles producing a match with a maternal allele.

\section{Discussion}

This study provides the first direct proof of occasional paternal inheritance in a parthenogenetic animal. Paternal inheritance was found at a low rate of $4.72 \%$ of offspring. Because the exact chromosomal position of the analyzed microsatellite markers was only partially known, cases of paternal inheritance may have been undetected. Hence, the observed rate of paternity may be underestimated. In three other offspring, we found mother-offspring differences at one locus only. However, in these cases, mutation cannot be ruled out, because

Table 1 Proportion of paternal inheritance per family

\begin{tabular}{|c|c|c|c|c|}
\hline \multirow{2}{*}{$\begin{array}{l}\text { Family } \\
\text { (maternal } \\
\text { ploidy) }\end{array}$} & \multirow[t]{2}{*}{ Total offspring } & \multicolumn{2}{|c|}{$\begin{array}{l}\text { Offspring with } \\
\text { paternal alleles }\end{array}$} & \multirow[t]{2}{*}{ Sex rate } \\
\hline & & Addition & Displacement & \\
\hline A $(3 x)$ & 24 & - & - & 0 \\
\hline B $(4 x)$ & 4 & 1 & - & 0.25 \\
\hline$C(3 x)$ & 44 & 4 & - & 0.091 \\
\hline $\mathrm{D}(3 x)$ & 156 & 3 & - & 0.019 \\
\hline $\mathrm{E}(3 x)$ & 6 & 1 & - & 0.167 \\
\hline $\mathrm{F}(3 x)$ & 62 & 1 & - & 0.016 \\
\hline $\mathrm{G}(3 x)$ & 28 & - & - & 0 \\
\hline$H(3 x)$ & 2 & - & - & 0 \\
\hline I $(3 x)$ & 10 & - & - & 0 \\
\hline $\mathrm{J}(3 x)$ & 6 & - & - & 0 \\
\hline $\mathrm{K}(3 x)$ & 39 & - & 8 & 0.205 \\
\hline Total & 381 & 10 & 8 & 0.047 \\
\hline
\end{tabular}

In five out of 11 families, no paternal inheritance was detected. In the other six families, $5.79 \%$ of the offspring showed incorporation of paternal alleles, either by addition of paternal alleles or displacement of maternal alleles. changes occurred at only one locus and/or consisted of small microsatellite size variation. Following a conservative interpretation of results, we classified these three cases as clonal offspring.

Two different mechanisms for the incorporation of paternal alleles have been detected, namely allele addition and allele displacement. As only one allele per locus is paternally added to the offspring and only one maternal allele per locus is lost (except supernumerary alleles of SpATT9), loss and addition of whole chromosome sets is likely. This conclusion is also supported by the fact that at least loci SpATT12 and SpATT20 are not linked (Pongratz et al, 2001) and aneuploidy has never been observed in $S$. polychroa. Consequently, allele addition coincides with increase of ploidy level $(3 x \rightarrow 4 x$, $4 x \rightarrow 5 x)$, whereas allele displacement results in identical mother-offspring ploidy $(3 x \rightarrow 3 x)$. These results confirm the mechanisms that we previously proposed to account for occasional sex in this parthenogenetic population (D'Souza et al, 2004).

We could not find evidence for a third mechanism (chromosome loss) in this study. This mechanism was previously indicated in tetraploid individuals, which produced triploid offspring at a rate of $\sim 12 \%$ (D'Souza et al, 2004). However, in the current study, only one tetraploid individual was fertile and produced only four offspring (family B). This number is clearly too small for a rigorous test of the occurrence of chromosome loss.

The mechanism restoring regular numbers of chromosomes (either $3 x, 4 x$ or rarely $5 x$ ) has still to be clarified. The chromosome displacement pathway, in particular, needs further study. Although the present data suggest displacement of whole chromosome sets, we cannot exclude the possibility that only some paternal chromosomes remain in the zygote. During asexual reproduction, paternal chromosomes either degenerate within the zygote or are expelled from the zygote with one of the polar bodies (Benazzi Lentati, 1970). Instead of paternal chromosomes, maternal ones may be sometimes excluded from the zygote by the same mechanisms. However, maternal chromosomes may also be eliminated before fusion with sperm during oogenesis. Irrespective of the exact mechanism, chromosome elimination and incorporation have to be cytogenetically adjusted, as otherwise aneuploid or diploid individuals may

Table 2 Two examples of families with paternal inheritance. For illustration purpose we displayed only the results of three microsatellite loci

\begin{tabular}{|c|c|c|c|c|c|c|c|c|c|c|c|c|c|c|c|c|}
\hline \multicolumn{2}{|c|}{ Family } & \multicolumn{5}{|c|}{ SpATT12 } & \multicolumn{6}{|c|}{ SpATT20 } & \multicolumn{4}{|c|}{ SpATT7 } \\
\hline $\mathrm{D}$ & q & & $\begin{array}{c}14 \\
\downarrow\end{array}$ & $\begin{array}{c}15 \\
\downarrow\end{array}$ & $\begin{array}{c}27+50 \\
\downarrow\end{array}$ & & $\begin{array}{c}23 \\
\downarrow\end{array}$ & & & $\begin{array}{c}34 \\
\downarrow\end{array}$ & $\begin{array}{c}43 \\
\downarrow\end{array}$ & & $\begin{array}{c}70 \\
\downarrow\end{array}$ & & $\begin{array}{c}73 \\
\downarrow\end{array}$ & \\
\hline & D4 (addition) & & 14 & 15 & $27+50$ & $\begin{array}{c}34+55 \\
\uparrow\end{array}$ & 23 & & $\begin{array}{c}33 \\
\uparrow\end{array}$ & 34 & 43 & & 70 & $\begin{array}{c}71 \\
\uparrow\end{array}$ & 73 & \\
\hline & $\hat{0}$ & 11 & & & & $34+55$ & & 24 & 33 & & 43 & & & 71 & 73 & \\
\hline \multirow[t]{3}{*}{ K } & q & $\begin{array}{c}11 \\
\downarrow\end{array}$ & $\begin{array}{c}12 \\
\downarrow\end{array}$ & & 15 & & $\begin{array}{c}23 \\
\downarrow\end{array}$ & 24 & & $\begin{array}{c}34 \\
\downarrow\end{array}$ & & & $\begin{array}{c}69 \\
\downarrow\end{array}$ & & 71 & \\
\hline & K2 (displacement) & 11 & 12 & & $x$ & $\begin{array}{c}27+50 \\
\uparrow\end{array}$ & 23 & $x$ & & 34 & $\begin{array}{c}42 \\
\uparrow\end{array}$ & & 69 & & $X$ & $\begin{array}{c}73 \\
\uparrow\end{array}$ \\
\hline & $\hat{0}$ & & & 14 & 15 & $27+50$ & 23 & & 33 & & 42 & 43 & & 70 & & 73 \\
\hline
\end{tabular}

All families and offspring genotypes are displayed in Supplementary Information. Different alleles are shown as the number of microsatellite repeat units. Arrows indicate inheritance of alleles, and bold numbers paternal alleles in offspring. Missing maternal alleles are illustrated as $X$. In Family D, a typical example of addition of paternal alleles is given: All maternal alleles and one paternal allele per locus were inherited to the offspring. In family $\mathrm{K}$, paternal inheritance was accompanied with loss of one maternal allele per locus. 
originate. However, in contrast to other planarians (Beukeboom et al, 1998), aneuploidy has never been observed in S. polychroa. Moreover, all populations in central and western Europe lack diploid individuals and consist of only polyploid parthenogens (Beukeboom et al, 1996; Pongratz et al, 2003).

Owing to the recent advances in molecular and cytogenetics, more and more examples of rare sex in supposedly clonal species have been discovered (eg Burt et al, 1996; Belshaw et al, 1999; Samadi et al, 1999; Belshaw and Quicke, 2003; Chapman et al, 2004; Bock et al, 2005). However, little information about the exact underlying mechanisms is available. The main reason for this is that the presence of occasional sex is often indirectly inferred from population genetic parameters, such as genetic diversity or heterozygosity (Samadi et al, 1999; Reusch et al, 2000; Chapman et al, 2004; Bock et al, 2005). Examples of mechanisms that have been proposed for rare sex in supposedly clonal species are central fusion automixis in some Hymenoptera (Beukeboom and Pijnacker, 2000; Belshaw and Quicke, 2003), meiotic recombination and nonmeiotic recombination in dandelions (Mertens King and Schaal, 1990; van Baarlen et al, 2000), or other covert forms of meiosis and segregation (Hurst et al, 1992).

Our studies demonstrate by direct parent-offspring comparison that sperm-dependent parthenogens possess two alternative mechanisms for occasional sex: chromosome displacement (one-generation cycle) and also chromosome addition $(3 x \rightarrow 4 x)$. If chromosome addition were to be followed by chromosomal loss $(4 x \rightarrow 3 x)$, as has been detected in $S$. polychroa, a unique twogeneration cycle of mixing of maternal and paternal chromosomes and sexual recombination would emerge (D'Souza et al, 2004).

Although the incidence of ploidy variation within parthenogenetic species and sperm dependency may be suggestive, genetic exchange between parthenogens has only been unequivocally demonstrated in the sperm-dependent dace Phoxinus eos-neogaeus (Goddard and Schultz, 1993; Goddard et al, 1998) and hermaphroditic dandelion Taraxacum (Verduijn et al, 2004). However, geneflow between these parthenogens is mediated by sexual sperm/pollen donors, which is in contrast with the case of $S$. polychroa, where chromosome sets are exchanged in exclusively parthenogenetic populations.

Theoretically, parthenogenetic populations are seen as evolutionary dead ends. However, parthenogenesis seems to be a stable reproductive mode in several species. This paradox may be explained by undetected mechanisms of sexual reproduction in supposedly clonal species (Hurst et al, 1992). Low rates of sex may help to maintain sufficient genetic variation, to enhance the incorporation of beneficial mutations (Pamilo et al, 1987; Peck, 1994; Green and Noakes, 1995) and slow down the accumulation of deleterious mutations (Pamilo et al, 1987; Green and Noakes, 1995; Haccou and Schneider, 2004). After only one sexual generation, most of the hidden genetic variance of asexuals is released with the consequence that deleterious mutations can efficiently be eliminated by natural selection (Lynch and Gabriel, 1983; Wagner and Gabriel, 1990). Occasional recombination also helps to stabilize complex host-parasite dynamics (Flatt et al, 2001) and allows for rapid evolution of resistance against coevolving parasites (Hamilton, 1980;
Lively and Howard, 1994). Moreover, under negative frequency-dependent selection, which may arise owing to host-parasite interactions, parthenogens with rare sex are able to invade obligate sexual and obligate asexual species (Yamauchi, 1999; Yamauchi and Kamite, 2003). In conclusion, rare sex in predominately clonally reproducing organisms could accrue the benefits of sex without paying its full costs (Hurst and Peck, 1996).

The sperm dependency observed in several parthenogenetic species could therefore be explained if it allows rare sex, and hence the combination of the advantages of sex and asex (Beukeboom and Vrijenhoek, 1998; Schlupp, 2005). Paternal leakage in sperm-dependent parthenogens has been found in the Amazon Molly Poecilia formosa (Schartl et al, 1995; Lamatsch et al, 2000) and Phoxinus eos-neogaeus (Goddard and Schultz, 1993; Goddard et al, 1998). Moreover, the occurrence of regular paternity may also explain why sperm-dependent hermaphrodites still invest in male organs, even though a parthenogen allocating all resources into the female function would be expected to easily outcompete hermaphroditic parthenogens. An understanding of the diversity and frequency of rare sexual processes in parthenogenetic species clearly warrants further investigation. Such studies should help to explain the distribution and persistence of parthenogenesis in natural populations.

\section{Acknowledgements}

We thank Ramona Wesselmann for the help in flatworm collection, Barbara Hasert and Gaby Niester for lab assistance, Maike de Buhr, Simone Riss and Gregor Schulte for observing flatworm matings, and David Mark Welch, Beatriz Sanchez Navarro, Koen Martens and an anonymous referee for suggestions to improve earlier drafts of the manuscript.

\section{References}

Belshaw R, Quicke DLJ (2003). The cytogenetics of thelytoky in a predominantly asexual parasitoid wasp with covert sex. Genome 46: 170-173.

Belshaw R, Quicke DLJ, Volkl W, Godfray HCJ (1999). Molecular markers indicate rare sex in a predominantly asexual parasitoid wasp. Evolution 53: 1189-1199.

Benazzi Lentati G (1970). Gametogenesis and egg fertilization in planarians. Int Rev Cytol 27: 101-179.

Beukeboom LW, Pijnacker LP (2000). Automictic parthenogenesis in the parasitoid Venturia canescens (Hymenoptera: Ichneumonidae) revisited. Genome 43: 939-944.

Beukeboom LW, Sharbel TF, Michiels NK (1998). Reproductive modes, ploidy distribution, and supernumerary chromosome frequencies of the flatworm Polycelis nigra (Platyhelminthes: Tricladida). Hydrobiologia 383: 277-285.

Beukeboom LW, Vrijenhoek RC (1998). Evolutionary genetics and ecology of sperm-dependent parthenogenesis. J Evol Biol 11: 755-782.

Beukeboom LW, Weinzierl RP, Reed KM, Michiels NK (1996). Distribution and origin of chromosomal races in the freshwater planarian Dugesia polychroa (Turbellaria: Tricladida). Hereditas 124: 7-15.

Bock CH, Thrall PH, Burdon JJ (2005). Genetic structure of populations of Alternaria brassicicola suggests the occurrence of sexual recombination. Mycol Res 109: 227-236.

Burt A, Carter DA, Koenig GL, White TJ, Taylor JW (1996). Molecular markers reveal cryptic sex in the human 
pathogen Coccidioides immitis. Proc Natl Acad Sci USA 93: 770-773.

Chapman H, Robson B, Pearson ML (2004). Population genetic structure of a colonising, triploid weed, Hieracium lepidulum. Heredity 92: 182-188.

D'Souza TG, Storhas M, Schulenburg H, Beukeboom LW, Michiels NK (2004). Occasional sex in an 'asexual' polyploid hermaphrodite. Proc R Soc London B 271: 1001-1007.

Flatt T, Maire N, Doebeli M (2001). A bit of sex stabilizes hostparasite dynamics. J Theor Biol 212: 345-354.

Goddard KA, Schultz RJ (1993). Aclonal reproduction by polyploid members of the clonal hybris species Phoxinuseos-neogaeus (Cyprinidae). Copeia 3: 650-660.

Goddard KA, Wessner LL, Giaimo F, Megwinoff O (1998). Confirmation of gynogenesis in Phoxinus eos-neogaeus (Pisces: Cyprinidae). J Hered 89: 151.

Gomez-Zurita J, Funk DJ, Vogler AP (2006). The evolution of unisexuality in Calligrapha leaf beatles: molecular and ecological insights on multiple origins via interspecific hybridization. Evolution 60: 328-347.

Green RF, Noakes DLG (1995). Is a little bit of sex as good as a lot? J Theor Biol 174: 87-96.

Haccou P, Schneider MV (2004). Modes of reproduction and the accumulation of deleterious mutations with multiplicative fitness effects. Genetics 166: 1093-1104.

Hamilton WD (1980). Sex versus non-sex versus parasite. Oikos 35: 282-290.

Hurst LD, Hamilton WD, Ladle RJ (1992). Covert sex. Trends Ecol Evol 7: 144-145.

Hurst LD, Peck JR (1996). Recent advances in understanding of the evolution and maintenance of sex. Trends Ecol Evol 11: 46-52.

Lamatsch DK, Nanda I, Epplen JT, Schmid M, Schartl M (2000). Unusual triploid males in a microchromosome-carrying clone of the Amazon molly, Poecilia formosa. Cytogenet Cell Genet 91: 148.

Lampert KP, Lamatsch DK, Epplen JT, Schartl M (2005). Evidence for a monophyletic origin of triploid clones of the Amazon molly, Poecilia formosa. Evolution 59: 881-889.

Lively CM, Howard RS (1994). Selection by parasites for clonal diversity and mixed mating. Philos Trans $R$ Soc London B 346: 271-281.

Lynch M, Gabriel W (1983). Phenotypic evolution and parthenogenesis. Am Nat 122: 745-764.

Mertens King L, Schaal BA (1990). Genotypic variation within asexual lineages of Taraxacum officinale. Proc Natl Acad Sci USA 87: 998-1002.

Michiels NK, Kuhl A (2003). Altruistic sperm donation in a sperm-dependent parthenogenetic hermaphrodite is stabilized by reciprocal sperm exchange. J Zool 259: 77-82.

Michiels NK, Streng A (1998). Sperm exchange in a simultaneous hermaphrodite. Behav Ecol Sociobiol 42: 171-178.

Otto SP, Lenormand T (2002). Resolving the paradox of sex and recombination. Nat Rev Genet 3: 252-261.

Pamilo P, Nei M, Li WH (1987). Accumulation of mutations in sexual and asexual populations. Genet Res 49: 135-146.

Parker ED (1979). Ecological implications of clonal diversity in parthenogenetic morphospecies. Am Zool 19: 753-762.
Peck JR (1994). A ruby in the rubbish - beneficial mutations, deleterious mutations and the evolution of sex. Genetics 137: 597-604

Pongratz N, Gerace L, Alganza AM, Beukeboom LW, Michiels NK (2001). Microsatellite development and inheritance in the planarian flatworm Schmidtea polychroa. Belg J Zool 131: 71-75.

Pongratz N, Storhas M, Carranza S, Michiels NK (2003). Phylogeography of competing sexual and parthenogenetic forms of a freshwater flatworm: patterns and explanations. BMC Evol Biol 3 (article no. 23).

Ramachandran S, Beukeboom LW, Gerace L, Pavlovic N, Carranza S, Michiels NK (1997). Isolation and characterization of microsatellite loci from the planarian Dugesia polychroa (Schmidt) (Platyhelminthes: Tricladida). Mol Ecol 6: 389-391.

Redi CA, Garagna S, Pellicciari C (1982). Chromosome preparation from planarian blastemas: a new procedure suitable for cytogenetic and cytochemical studies. Stain Technol 57: 190-192.

Reusch TBH, Stam WT, Olsen JL (2000). A microsatellitebased estimation of clonal diversity and population subdivision in Zostera marina, a marine flowering plant. Mol Ecol 9: $127-140$.

Samadi S, Mavarez J, Pointier JP, Delay B, Jarne P (1999). Microsatellite and morphological analysis of population structure in the parthenogenetic freshwater snail Melanoides tuberculata: insights into the creation of clonal variability. Mol Ecol 8: 1141-1153.

Schartl M, Nanda I, Schlupp I, Wilde B, Epplen JT, Schmid M et al (1995). Incorporation of subgenomic amounts of DNA as compensation for mutational load in a gynogenetic fish. Nature 373: 68-71.

Schlupp I (2005). The evolutionary ecology of gynogenesis Annu Rev Ecol Evolut Syst 36: 399-417.

Schön I, Butlin RK, Martens K, Gandolfi A, Di Masso E, Rossi V et al (2000). Persistence of asexuality through mixed reproduction in Eucypris virens (Crustacea, Ostracoda). Heredity 84: 161-169.

Storhas M, Weinzierl RP, Michiels NK (2000). Paternal sex in parthenogenetic planarians: a tool to investigate the accumulation of deleterious mutations. J Evol Biol 13: 1-8.

van Baarlen P, van Dijk P, Hoekstra RF, de Jong JH (2000). Meiotic recombination in sexual diploid and apomictic triploid dandelions (Taraxacum officinale L.) 146. Genome 43: 827-835.

Verduijn MH, Van Dijk PJ, Van Damme JMM (2004). The role of tetraploids in the sexual-asexual cycle in dandelions (Taraxacum). Heredity 93: 390-398.

Wagner GP, Gabriel W (1990). Quantitative variation in finite parthenogenetic populations: what stops Muller's ratchet in the absence of recombination? Evolution 44: 715-731.

West SA, Lively CM, Read AF (1999). A pluralist approach to sex and recombination. J Evol Biol 12: 1003-1012.

Yamauchi A (1999). Evolution of cyclic sexual reproduction under host-parasite interactions. J Theor Biol 201: 281.

Yamauchi A, Kamite Y (2003). Facultative sexual reproduction under frequency-dependent selection on a single locus. J Theor Biol 221: 411-424.

Supplementary Information accompanies the paper on Heredity website (http://www.nature.com/hdy) 